\title{
Efeitos da Climatização do Curral de Espera na Produção de Leite de Vacas Holandesas
} Iran J.O. da Silva1 ${ }^{1}$, Héliton Pandorfi ${ }^{2}$, Irineu Acararo Jr. ${ }^{3}$, Sônia M.S. Piedade ${ }^{4}$, Daniella Jorge de Moura ${ }^{5}$

\footnotetext{
RESUMO - O presente estudo foi conduzido com o objetivo de avaliar os efeitos da climatização de ambientes em condições de pré-ordenha na produção de leite. O experimento foi realizado na fazenda experimental do Instituto de Zootecnia, em Nova Odessa, SP, durante doze dias consecutivos do verão de 1999. Foram analisados os efeitos do sistema de resfriamento evaporativo (SRE), por nebulização, no curral de espera. Utilizaram-se doze animais da raça holandesa, divididos em dois grupos de seis animais, onde apenas o segundo grupo foi submetido ao sistema de climatização. Os dados ambientais foram registrados no horário das ordenhas, ou seja, às 7 e 15 h. Para avaliação dos dados ambientais utilizaram-se os índices de conforto térmico: índice de temperatura de globo (WBGT), índice de temperatura e umidade (THI) e índice de carga térmica radiante (CTR). As respostas fisiológicas, como freqüência cardíaca e respiratória, temperatura retal e produção de leite também foram consideradas variáveis de resposta. Conclui-se que o efeito do resfriamento do ambiente é mais significativo no horário da segunda ordenha (15 h), quando comparado ao da primeira (7 h), melhorando as condições de conforto térmico no curral de espera, refletindo em um aumento de 7,28\% na produção de leite, além de promover redução na frequência respiratória e cardíaca.
}

Palavras-chave: conforto térmico, leite, resfriamento evaporativo

\section{Effects of the Climatization of the Corral of Wait on Milk Production of Holstein Cows}

\begin{abstract}
This work was carried out to evaluate the effect of the climatization of environments in daily pay-milking conditions. The experiment was carried out in the experimental farm of the Institute of Zootecnia, in Nova Odessa, SP, during twelve consecutive days of the summer. The effects of the system of evaporative cooling (SRE), by fogging in the wait corral and milking room were analyzed. Twelve Holstein cows were divided in two groups, where only the second group had passed climatization. Data environment were recorded at 7:00 am and 3:00 pm. For evaluation of the ambient data, index of thermal comfort, index of globe temperature (WBGT), temperature humidity index (THI) and index of radiant thermal load (CTR) were used. The physiolological responses as cardiac frequency, respiratory frequency, retal temperature and production had been also considered variable responses. It was concluded that the effect of cooling of the environment is more significant in the schedule of second milking (3:00 pm) when compared to the of the first one (7:00 am), improving the conditions of thermal comfort in the wait corral, contemplating in an increase of 7,28\% in the production of milk, besides promoting a reduction in the breathing and heart frequency.
\end{abstract}

Key Words: thermal comfort, milk, evaporative cooling

\section{Introdução}

Dentre os problemas estratégicos ligados à produção animal, encontra-se o projeto das instalações para o confinamento dos animais. Em alguns casos, este item pode ser responsável pelo insucesso do sistema produtivo, sendo assim, de fundamental importância o estudo do ambiente interno destes abrigos. O animal é uma máquina biológica que deve expressar todo o seu potencial quando trabalha sobre ótimas condições ambientais. Quando estas condições não são adequadas, geralmente ocorre um reflexo negativo na produtividade.

Verifica-se a necessidade de desenvolver ainda mais a pecuária leiteira nos trópicos, onde muitas vezes a produção de leite é muito baixa. O problema principal está na adaptação das raças leiteiras, de origem européia, que devido a sua alta produtividade, muitas vezes sofrem problemas de alterações fisiológicas e de comportamento provocadas por estresse térmico, causando redução na produção de leite.

As alternativas para manter ou melhorar o desempenho e o bem-estar dos animais em climas quentes, necessariamente, envolvem a metabolização de energia e sua liberação para o ambiente, por meio dos processos de trocas térmicas entre o animal e o ambiente (Ingram, 1978; Johnson, 1987).

\footnotetext{
1 Prof. Dr. NUPEA (Núcleo de Pesquisa em Ambiência). Departamento de Engenharia Rural - ESALQ/USP. Cx. 09 - 13418-900. Piracicaba, SP. E.mail: ijosilva@carpa.ciagri.usp.br

${ }^{2}$ Engenheiro-Agrônomo. Pesquisador - NUPEA - ESALQ/USP, E.mail: hpandorf@esalq.usp.br

3 Pesquisador Científico. Instituto de Zootecnia (IZ) - Nova Odessa, SP.

${ }^{4}$ Profa. Dra. Departamento de Ciências Exatas - ESALQ - USP.

5 Profa. Dra. NUPEA (Núcleo de Pesquisa em Ambiência). Departamento de Engenharia Rural -ESALQ/USP.E.mail: djmoura@esalq.usp.br.
} 
Construções zootécnicas que possuem equipamentos de refrigeração e ventilação projetados especialmente para reduzir a temperatura do ar em abrigos para animais podem ser bastante eficientes. $\mathrm{O}$ resfriamento evaporativo das instalações, particularmente para o confinamento de gado leiteiro, tem se expandido rapidamente em climas quentes, devido a sua relativa simplicidade e praticidade técnica e à relação custo/benefício favorável, proporcionado incrementos na produção de leite.

O principal fator a ser considerado para se garantir o conforto ao animal em países tropicais e subtropicais é o de minimizar os efeitos do estresse térmico. As condições climáticas nessas regiões são um grande desafio aos produtores por alterarem os três processos vitais dos animais: a mantença, a reprodução e a produção de leite (Head, 1995).

De acordo com Rasgdale (1961), a temperatura ótima de produção de leite depende da espécie, raça e grau de tolerância ao calor e ao frio. A raça Holandesa tem sua produção reduzida a partir de $24^{\circ} \mathrm{C}$, a Suíça e a Jersey a partir de $27^{\circ} \mathrm{C}$ e as Zebuínas acima de $32^{\circ} \mathrm{C}$.

De modo geral, a zona de termoneutralidade (ZTN) de vacas Holandesas, em lactação, em termos de temperatura do ar, varia entre 4 e $26^{\circ} \mathrm{C}$ (Huber, 1990). A temperatura crítica superior para vacas em lactação Pardas Suíças e Jersey pode chegar a 27 e $29^{\circ} \mathrm{C}$, respectivamente (Hafez, 1975). Enquanto que para vacas Zebu, a crítica superior varia entre 30 e $35^{\circ} \mathrm{C}$ (Bianca, 1965).

A perda em produção de leite devido a altas temperaturas também depende da umidade relativa, velocidade do vento, e de outros fatores de manejo e alimentação. Essa perda pode atingir $10 \%$ ou mais quando comparadas com produções de animais que estão submetidos a ambientes climatizados (Head, 1995).

Vacas em lactação na Califórnia, no verão de 1977, experimentaram estresse térmico severo por um período de quase uma semana. Durante esse período, as máximas de temperatura diárias atingiram valores que variaram de 31 a $35,5^{\circ} \mathrm{C}$ e as mínimas de $21^{\circ} \mathrm{C}$. A umidade relativa do ar, média, variou entre 90 e $95 \%$. Aproximadamente 700 animais morreram devido ao estresse pelo calor e a produção de todas as vacas foi substancialmente reduzida (Buffington et al., 1983).

Dentro deste contexto, com o objetivo de reduzir a temperatura do ar ambiente favorecendo as trocas

R. Bras. Zootec., v.31, n.5, p.2036-2042, 2002 sensíveis de calor, o sistema de resfriamento evaporativo (SRE) visa aumentar a dissipação de calor na forma evaporativa e convectiva; muitos trabalhos de pesquisa mostraram os benefícios (devido o aumento de consumo de alimento e conseqüente aumento de produção) no uso de sistemas de chuveiros ou aspersores sob o animal, associados à ventilação forçada de ar (Bucklin et al., 1991). Esse sistema é bastante eficaz porque aumenta a capacidade de perda de calor por meio de evaporação e resfriamento; além disso ele pode ser posicionado em vários locais das instalações: sala de espera (antes da ordenha), área de alimentação, áreas de sombra, etc.

O objetivo desse estudo foi avaliar os efeitos da climatização de ambientes em condições de préordenha na produção de leite.

\section{Material e Métodos}

O estudo foi realizado na estação experimental do Instituto de Zootecnia, situado no município de Nova Odessa - SP, a $550 \mathrm{~m}$ de altitude, latitude 22042' S e longitude $47 \mathrm{o} 18^{\prime} \mathrm{W}$, numa propriedade rural, de produção intensiva de leite tipo B.

O experimento foi realizado durante 12 dias consecutivos no período de verão de 1999 , nos meses de janeiro e fevereiro.

Nesse experimento foram avaliadas as condições climáticas no curral de espera, ressaltando que o curral de espera e a sala de ordenha fazem parte de um mesmo prédio. Dessa forma os tratamentos adotados foram:

1. Curral de espera sem o sistema (SSRE).

2. Curral de espera com sistema de resfriamento evaporativo (SRE).

O ambiente estudado foi adaptado com dois ventiladores, fabricado por Kohlbach Motores Ltda, motor de indução trifásico número 895 , modelo 48 , categoria $\mathrm{N}$, com $0,75 \mathrm{cv}$ e $3400 \mathrm{RPM}$, acoplados com umidificadores localizados a $2 \mathrm{~m}$ do solo, instalados no eixo transversal do curral de espera sob a tesoura do telhado.

Foram selecionados doze animais para o experimento, divididos em dois grupos de seis vacas da raça holandesa, com produção média de leite de 17,5 litros, sendo distribuídas por critérios de pureza, ordem de parto e estádio de lactação, garantido a maior homogeneidade entre os dois grupos.

Durante o estudo, os animais permaneceram no curral de espera por determinado tempo. O primeiro 
grupo, sem o sistema de resfriamento (tratamento 1), foi ordenhado imediatamente, porém, o segundo grupo (tratamento 2) permaneceu no curral de espera sob a climatização durante aproximadamente $40 \mathrm{mi}-$ nutos, período para que todo o lote fosse ordenhado. O tempo de espera, foi adotado de acordo com o manejo da unidade leiteira.

Durante todo o experimento foram monitoradas as variáveis ambientais do curral de espera por meio de um termohigrômetro e termômetro de globo negro. Foram registradas temperatura máxima (Tmáx.), temperatura mínima (Tmín.), temperatura de bulbo seco (TBS), temperatura de bulbo úmido (TBU), umidade relativa (UR\%), temperatura de globo negro (TG), no horário das 7 e $15 \mathrm{~h}$, durante as ordenhas do dia. Os dados foram correlacionados para a avaliação pelos índices de conforto térmico. Os índices utilizados nessa análise de dados foram:

Índice de Temperatura de Globo (WBGT), proposto por Bond \& Kelly (1955):

$\mathrm{WBGT}=0,7 \mathrm{TBU}+0,2 \mathrm{TG}+0,1 \mathrm{TA}$

em que: $\mathrm{TBU}=$ temperatura de bulbo úmido $\left({ }^{\circ} \mathrm{C}\right)$; $\mathrm{TG}=$ temperatura de globo $\left({ }^{\circ} \mathrm{C}\right) ; \mathrm{TA}=$ temperatura ambiente $\left({ }^{\circ} \mathrm{C}\right)$.

Índice de Temperatura e Umidade (THI), desenvolvido por THOM (1958):

$$
\mathrm{THI}=\mathrm{TBS}+0,36 \mathrm{TBU}+41,5
$$

em que: $\mathrm{TBS}=$ temperatura de bulbo seco $\left({ }^{\circ} \mathrm{C}\right)$; TBU $=$ temperatura de bulbo úmido $\left({ }^{\circ} \mathrm{C}\right)$.

Índice de Carga Térmica Radiante (CTR), proposto por ESMAY (1979):

$$
\begin{gathered}
\text { CTR }=\mathrm{t}(\mathrm{TMR}) 4 \\
\mathrm{TMR}=100((2.51(\mathrm{VV}) 05(\mathrm{TG}-\mathrm{Ta})+ \\
(\mathrm{TG} / 100) 4) 0.25)
\end{gathered}
$$

em que: TRM = temperatura média radiante; $\mathrm{VV}=$ velocidade do vento $(\mathrm{m} / \mathrm{s}) ; \mathrm{TG}=$ temperatura de globo negro $(\mathrm{K})$; $\mathrm{Ta}=$ temperatura ambiente $(\mathrm{K})$; $\mathrm{t}=5.67 * 10-8 \mathrm{~K}-4 . \mathrm{W} / \mathrm{m}^{2}$ (Constante de StefanBoltzmann).

A adoção de vários índices de conforto nesse estudo foi para verificar a tendência nas respostas físicas do ambiente, ou seja, alguns índices são mais sensíveis à temperatura de globo negro (WBGT), à ventilação natural (CTR) dos ambientes, e outro à depressão psicrométrica como o caso do THI.

Em função das comparações, verificou-se a possibilidade de as respostas serem semelhantes para os diferentes índices.

Os parâmetros utilizados para avaliação fisiológica restringiram-se à monitoração da temperatura retal, frequência respiratória e cardíaca, que foram registrados nos animais na chegada ao curral de espera e na saída da sala de ordenha. O registro desses dados foram realizados diariamente, em três animais de cada tratamento, primeiramente escolhidos de forma aleatória, mas permanecendo fixos durante todo o experimento.

O delineamento experimental adotado foi o inteiramente casualizado (DIC), adotando como tratamentos a não climatização e a climatização do curral de espera e as repetições, o número de animais estudados no experimento. As variáveis respostas de produção, e as ambientais foram analisadas adotando o DIC para análise geral, e o teste de Tukey $(\mathrm{P}<0,05)$ para a comparação entre as médias. Foi feita a análise para cada horário (7 e 15 h) e período do estudo (12 dias consecutivos), sendo que os dados ambientais coletados e os índices de conforto calculados obedeceram o seguinte modelo matemático:

$$
Y_{i j}=\mu+b_{i}+b_{j}+b_{k}+e i j
$$

em que: $Y_{i j}=$ média da variável ambiental; $\mu$ = média geral da variável ambiental; $b_{i}=$ efeito dos tratamentos utilizados; $b_{j}=$ efeito dos dias estudados na variável ambiental; $b_{k}=$ efeito do horário na variável meteorológica; eij = erro aleatório.

O mesmo procedimento foi feito entre os dados fisiológicos, temperatura retal, freqüência cardíaca e respiratória, adotando-se o programa estatístico Statistical Analysis Sistem (SAS, 1992), para análise dos dados.

\section{Resultados e Discussão}

Na Tabela 1, são apresentados os resultados finais das médias das variáveis respostas na primeira ordenha, realizada às $7 \mathrm{~h}$. Verificou-se que os tratamentos com e sem nebulização apresentaram comportamentos diferentes no curral de espera. $\mathrm{O}$ ambiente climatizado com Sistema de Resfriamento Evaporativo (SRE) no curral de espera diferiu $(\mathrm{P}<0,05)$ daquele sem o sistema de resfriamento somente quando se usou o índice de temperatura e umidade (THI). Porém, as diferenças nas variáveis ambientais, Ta e TG, não apresentaram diferenças significativas. Esse fato é atribuído às condições climáticas externas, pois neste horário a temperatura é mais amena, o que não evidência o efeito da nebulização nas variáveis climáticas. Mesmo o efeito na UR\% dos ambientes foram evidenciados.

Com relação aos índices de conforto térmico, 
Tabela 1 - Valores médios das variáveis ambientais e dos índices de conforto observados durante a ordenha das $7 \mathrm{~h}$

Table 1 - Average values of the environment traits and comfort index observed during milking at 7:00 am

\begin{tabular}{lcc}
\hline & \multicolumn{2}{c}{$1^{\mathrm{a}}$ ordenha (curral de espera) } \\
Variáveis & \multicolumn{1}{c}{ milking (wait corral) $^{\text {st neb. }}{ }^{1}$} & $\mathrm{~s} /$ neb. \\
ambientais & with evaporative & without evaporative \\
Environmental & cooling & cooling \\
traits & & \\
\hline $\mathrm{TG}^{3}$ & $22,61^{\mathrm{a}}$ & $22,54^{\mathrm{a}}$ \\
$\mathrm{Ta}^{4}$ & $23,22^{\mathrm{a}}$ & $24,30^{\mathrm{a}}$ \\
$\mathrm{UR}^{5}$ & $96,50^{\mathrm{a}}$ & $83,50^{\mathrm{b}}$ \\
$\mathrm{THI}^{6}$ & $72,92^{\mathrm{b}}$ & $73,76^{\mathrm{a}}$ \\
$\mathrm{WBGT}^{7}$ & $22,73^{\mathrm{a}}$ & $22,25^{\mathrm{a}}$ \\
$\mathrm{CTR}^{8}$ & $422,59^{\mathrm{a}}$ & $424,22^{\mathrm{a}}$ \\
\hline
\end{tabular}

${ }^{1}$ com nebulização (with evaporative cooling), ${ }^{2}$ sem nebulização (without evaporative cooling), ${ }^{3}$ TG (temperatura de globo negro) (black globe temperature), ${ }^{4} \mathrm{Ta}$ (temperatura do ar) - (air temperature), ${ }^{5} \mathrm{UR} \%$ (umidade relativa) - (humidity relative), ${ }^{6} \mathrm{THI}$ (índice de temperatura e umidade) - (temperature humidity index), ${ }^{7}$ WBGT (índice de temperatura de globo) - (index of globe temperature), ${ }^{8}$ CTR (índice de carga térmica radiante) - (index of radiant thermal load).

Médias na mesma linha, seguidas pela mesma letra, não diferem pelo teste de Tukey a $5 \%$ de probabilidade (Means, within a row, followed by the same letter, do not differ at $5 \%$ of probability by Tukey test).

verificou-se que às $7 \mathrm{~h}$ o ambiente apresentou-se em condições ideais, $75 \geq \mathrm{THI} \geq 72$.

Na comparação entre os índices verificou-se que no horário das $7 \mathrm{~h}$, os efeitos convectivos no ambiente não foram significativos, o que pode ser observado nas análises da CTR e do WBGT.

Com relação às respostas fisiológicas, como freqüência cardíaca e temperatura retal, não se observou diferença significativa entre as médias apresentadas na Tabela 2. No entanto, a frequiência respiratória foi $18,24 \%$ inferior $(\mathrm{P}<0,05)$ para o grupo submetido à climatização, em relação àquela do grupo controle.

Constata-se na Figura 1 o efeito do aumento da produção nos animais submetidos à climatização no curral de espera. Nessas condições, verificou-se aumento $(\mathrm{P}<0,05)$ médio de $5,19 \%$ na produção de leite (Tabela 2), no grupo de animais que foram expostos ao curral de espera com climatização em relação ao controle.

Verifica-se na Figura 2 que ao nebulizar o curral de espera, alterações significativas de redução ocorrem na freqüência respiratória. Esses resultados condizem com o trabalho de Tinôco (1988), que verificou a eficiência do processo de resfriamento evaporativo, pois as condições climáticas desfavoráveis em um ambiente, causam alterações nos
Tabela 2 - Valores médios de produção e respostas fisiológicas dos animais obtidos no período da ordenha das $7 \mathrm{~h}$

Table 2 - Average values of production and physiologic responses observed during milking at 7:00 am

$1^{\text {a }}$ ordenha (curral de espera) $1^{\text {st }}$ milk (wait corral)

Variáveis

ambientais

Environmental c/ neb. ${ }^{1} \quad$ s/ neb. ${ }^{2}$

traits

\begin{tabular}{lcc}
\hline $\begin{array}{l}\text { Produção } \\
\text { Production }\end{array}$ & $12,13^{\mathrm{a}}$ & $11,50^{\mathrm{b}}$ \\
$\begin{array}{l}\text { Frequência cardíaca } \\
\text { Heartfrequency }\end{array}$ & $70,66^{\mathrm{a}}$ & $79,50^{\mathrm{a}}$ \\
$\begin{array}{l}\text { Frequência respiratória } \\
\text { Respiratoryfrequency }\end{array}$ & $53,00^{\mathrm{b}}$ & $62,66^{\mathrm{a}}$ \\
$\begin{array}{l}\text { Temperatura retal } \\
\text { Rectal temperature }\end{array}$ & $38,35^{\mathrm{a}}$ & $38,48^{\mathrm{a}}$ \\
\end{tabular}

${ }^{1}$ com nebulização (with evaporative cooling), ${ }^{2}$ sem nebulização (without evaporative cooling).

Médias na mesma linha, seguidas pela mesma letra, não diferem pelo teste de Tukey a $5 \%$ de probabilidade (Means, within a row, followed by the same letter, do not differ at $5 \%$ of probability by Tukey test).

batimentos cardíacos, frequiência respiratória e temperatura retal dos animais. Sabe-se que o sistema adotado causa variações na temperatura do ambiente, consequentemente as respostas fisiológica e de produção também serão alteradas em função das condições microclimáticas.

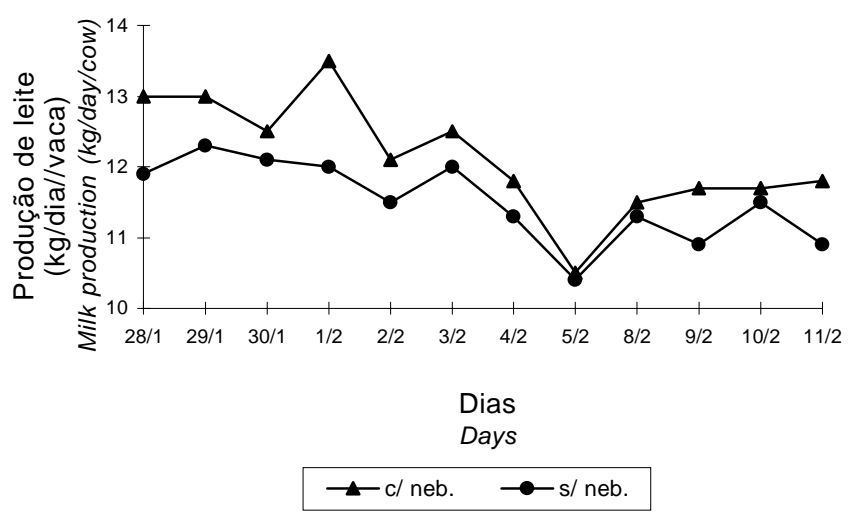

Figura 1 - Variação da produção de leite, em animais expostos no curral de espera com nebulização (c/neb.) e sem nebulização (s/neb.) na ordenha das $7 \mathrm{~h}$.

Figure 1 - Variation of the milk production, in animals displayed in the corral of wait with evaporative cooling (c/neb.) e without evaporative cooling ( $s$ / neb.) in it milks of the 7:00 am. 


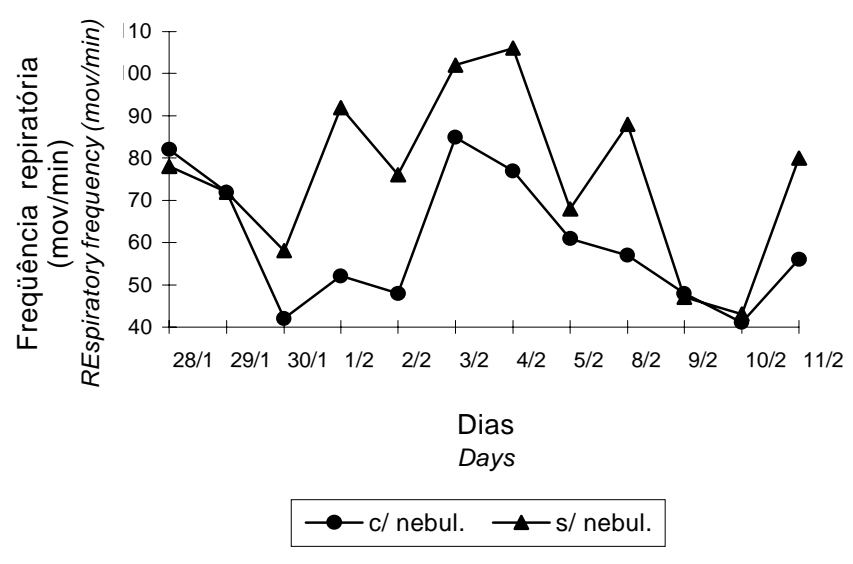

Figura 2 - Variação da freqüência respiratória dos animais expostos em ambientes com nebulização (c/nebul.) e sem nebulização (s/nebul.) na ordenha das $7 \mathrm{~h}$.

Figure 2 - Variation of the respiratory frequency of the animals under environments with evaporative cooling (c/nebul.) and without evaporative cooling (s/nebul.) during milking at 7:00 am.
Tabela 3 - Valores médios das variáveis ambientais e dos índices de conforto observados durante a ordenha das $15 \mathrm{~h}$

Table 3 - Average values of the environment traits and comfort index observed during milking at 3:00 pm

\begin{tabular}{lcc}
\hline & \multicolumn{2}{c}{$2^{\mathrm{a}}$ ordenha (curral de espera) } \\
Variáveis & \multicolumn{2}{c}{$2^{\text {nd }}$ milking (wait corra) } \\
\cline { 2 - 3 } ambientais & $\mathrm{c} /$ neb. & s/ neb. \\
Environmental & with evaporative & without evaporative \\
traits & cooling & cooling \\
TG $^{3}$ & & \\
Ta $^{4}$ & $24,87^{\mathrm{b}}$ & $27,23^{\mathrm{a}}$ \\
$\mathrm{UR}^{5}$ & $25,22^{\mathrm{b}}$ & $27,75^{\mathrm{a}}$ \\
THI $^{6}$ & $95,83^{\mathrm{a}}$ & $79,66^{\mathrm{b}}$ \\
$\mathrm{WBGT}^{7}$ & $75,59^{\mathrm{b}}$ & $78,17^{\mathrm{a}}$ \\
CTR $^{8}$ & $24,72^{\mathrm{a}}$ & $25,49^{\mathrm{a}}$ \\
\hline
\end{tabular}

${ }^{1}$ Com nebulização (with evaporative cooling), ${ }^{2}$ sem nebulização (without evaporative cooling), ${ }^{3} \mathrm{TG}$ (temperatura de globo negro) (black globe temperature), ${ }^{4} \mathrm{Ta}$ (temperatura do ar) (air temperature), ${ }^{5} \mathrm{UR} \%$ (umidade relativa) (relativehumidity), ${ }^{6} \mathrm{THI}$ (índice de temperatura e umidade) (temperature and humidity index), ${ }^{7}$ WBGT (índice de temperatura de globo) (index of globe temperature), ${ }^{8} \mathrm{CTR}$ (índice de carga térmica radiante) (index of radiant thermal load).

Médias na mesma linha, seguidas pela mesma letra, não diferem pelo teste de Tukey a $5 \%$ de probabilidade (Means, within a row, followed by the same letter, do not differ at $5 \%$ of probability by Tukey test).

$10 \%$ na produção de leite com animais submetidos a ambientes climatizados.

A variação da freqüência respiratória na ordenha das 15 h é nitidamente observada na Figura 4, na qual

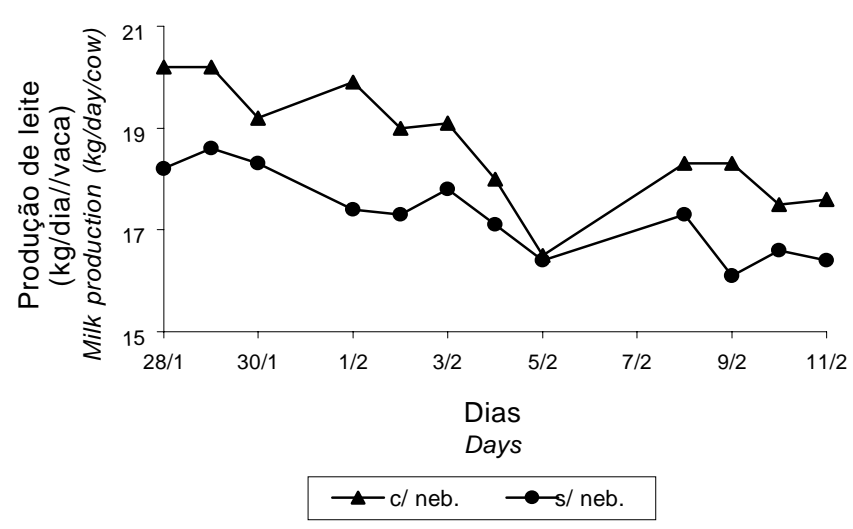

Figura 3 - Variação da produção diária de leite para animais expostos no curral de espera com nebulização (c/neb.) e sem nebulização (s/neb.) na ordenha das 7 e $15 \mathrm{~h}$.

Figure 3 - Variation daily production of the milk for animals displayed in the corral of wait with evaporative cooling (c/neb.) and without evaporative cooling (s/neb.) in it milks of the 7:00 am and 3:00 pm. na produção de leite, condizendo com os resultados obtidos por Head (1995), onde se atingiu aumento de

\section{R. Bras. Zootec., v.31, n.5, p.2036-2042, 2002}


Tabela 4 - Valores médios de produção e respostas fisiológicas dos animais obtidos no período da ordenha das $15 \mathrm{~h}$

Table 4 - Average values of production and physiologic responses of the animals observed during milking at 3:00 pm

\begin{tabular}{|c|c|c|}
\hline \multirow[b]{2}{*}{$\begin{array}{l}\text { Variáveis } \\
\text { ambientais } \\
\text { Environmental } \\
\text { traits }\end{array}$} & \multicolumn{2}{|c|}{$\begin{array}{l}2^{\mathrm{a}} \text { ordennha (Curral de espera) } \\
2^{\text {nd }} \text { milking (wait corra) }\end{array}$} \\
\hline & $\begin{array}{l}\mathrm{c} / \text { neb. }{ }^{1} \\
\text { with evaporative } \\
\text { cooling }\end{array}$ & $\begin{array}{c}\text { s/ neb. }{ }^{2} \\
\text { without evaporative } \\
\text { cooling }\end{array}$ \\
\hline $\begin{array}{l}\text { Produção } \\
\text { Production }\end{array}$ & $6,51^{\mathrm{a}}$ & $5,78^{b}$ \\
\hline $\begin{array}{l}\text { Frequência cardíaca } \\
\text { Cardiacfrequency }\end{array}$ & $73,42^{b}$ & $83,25^{\mathrm{a}}$ \\
\hline $\begin{array}{l}\text { Frequência respiratória } \\
\text { Respiratory frequency }\end{array}$ & $60,08^{b}$ & $75,83^{\mathrm{a}}$ \\
\hline $\begin{array}{l}\text { Temperatura retal } \\
\text { Retal temperature }\end{array}$ & $38,90^{\mathrm{a}}$ & $39,15^{\mathrm{a}}$ \\
\hline
\end{tabular}

${ }^{1}$ Com nebulização (with evaporative cooling).

2 Sem nebulização (without evaporative cooling).

Médias na mesma linha, seguidas pela mesma letra, não diferem pelo teste de Tukey a $5 \%$ de probabilidade (Means, within a row, followed by the same letter, do not differ at $5 \%$ of probability by Tukey test).

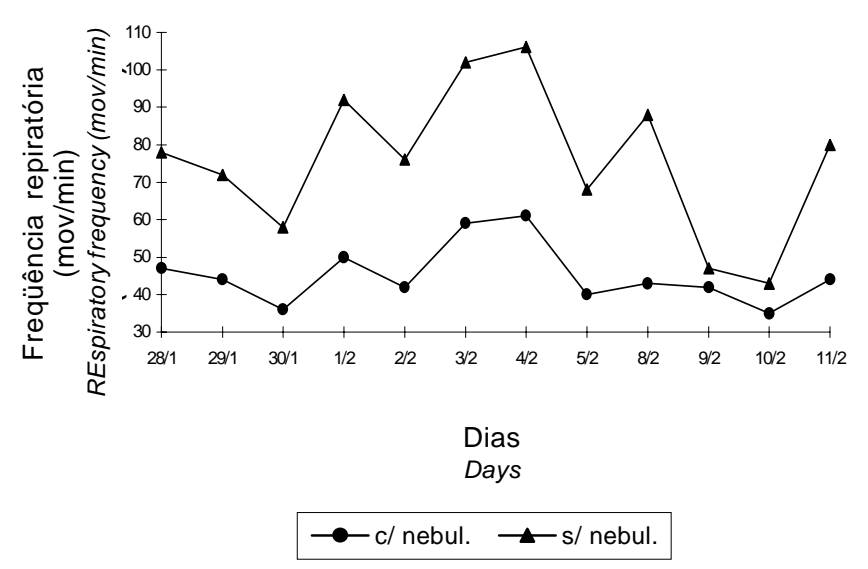

Figura 4 - Variação da freqüência respiratória dos animais expostos em ambientes com nebulização (c/nebul.) e sem nebulização (s/ nebul.) na ordenha das 15:00 horas.

Figure 4 - Variation of the respiratory frequency of the animals displayed in environments with evaporative cooling (c/nebul.) and without evaporative cooling (s/nebul.) in it milks of the 3:00 pm.

R. Bras. Zootec., v.31, n.5, p.2036-2042, 2002 se verifica que o uso de sistema de resfriamento evaporativo (SRE) reduz em média $26,21 \%$ da freqüência respiratória dos animais, bem como os batimentos cardíacos, que apresentaram um decréscimo de aproximadamente 13,38\% em média (Figura 5).

De acordo com os princípios de ambiência, pode-se inferir que, quando o animal está fora da sua zona termoneutra, qualquer mudança nas condições ambientais, por meio da climatização, poderá influenciar as suas características fisiológicas, e também de produção. Sabe-se que alterando as condições de temperatura e umidade relativa, modificam-se as condições de conforto, que terão influência direta nos índices de conforto térmico (Figura 6) e, conseqüentemente, na produção final do plantel (Figura 3).

Os resultados de alterações no microclima gerado, no interior do curral de espera, podem ser verificados pelo comportamento do índice THI (Figura 6). Notase que quando o ambiente é climatizado o índice THI se reduz, quando comparado com o ambiente sem climatização, apesar de não atingir os níveis de conforto adequado, essa redução promove uma condição considerada não crítica ao conforto térmico animal, resultando na minimização das perdas na produção de leite. Em geral , vacas holandesas em lactação apresentam declínio na produção de leite a partir de um THI igual a 72 (Johnson, 1980), garantindo a importância e eficiência do sistema de resfriamento empregado.

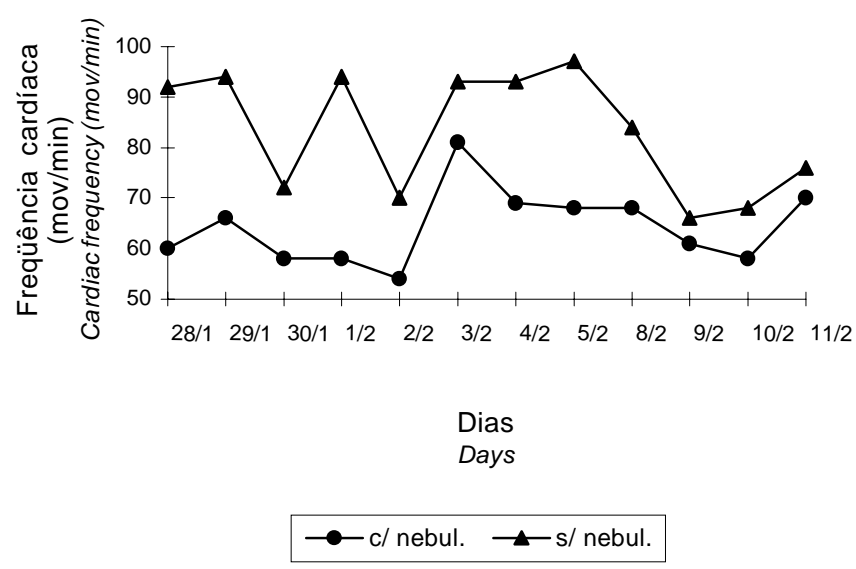

Figura 5 - Variação da freqüência cardíaca dos animais expostos em ambientes com nebulização (c/ nebul.) e sem nebulização (s/nebul.) na ordenha das 15:00 horas.

Figure 5 - Variation of the cardiac frequency of the animals displayed in environments with evaporative cooling (c/nebul.) and without evaporative cooling (s/nebul.) in it milks of the 3:00 pm. 


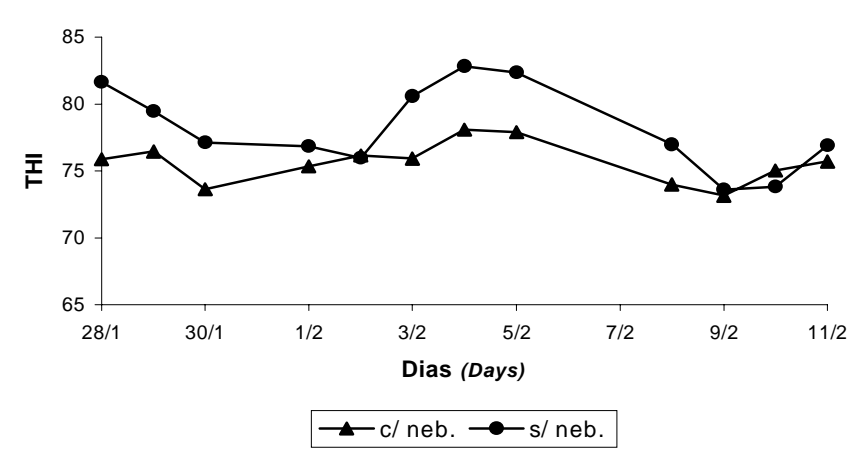

Figura 6 - Variação do índice de temperatura e umidade relativa do ambiente com nebulização (c/neb.) e sem nebulização (s/neb.) na ordenha das $15 \mathrm{~h}$.

Figure 6 - Variation of the index of temperature and relative humidity of the environment with evaporative cooling (c/neb.) and without evaporative cooling (s/neb.) during milking at 3:00 pm.

\section{Conclusões}

De acordo com as condições com que este experimento foi conduzido, conclui-se que a climatização de ambientes usando sistema de resfriamento adiabático evaporativo (SRAE) por nebulização na pré-ordenha, proporcionou um aumento na produção de leite de vacas da raça holandesa.

\section{Agradecimento}

Ao Instituto de Zootecnia de Nova Odessa - SP, por ceder a área experimental para execução do trabalho, e à Michela Maria de Paulo, pela revisão do conteúdo literário da publicação.

\section{Literatura Citada}

BIANCA, W. Reviews of the progress of dairy science. Section A. Physiology of cattle in hot environment. Journal of Dairy Research, v.32, n.1, p.291-245, 1965

BOND, T.E.; KELLY, C.F. The globe thermometer in agriculture research. Agricultural Engineer, v.36, n.2, p.251-260, 1955.

BUCKLIN, R.A.; BEEDE D.K.; BRAY, D.R. Methods to relieve heat stress for dairy cows in hot, humid climates. Applied Engineer Agricultural, v.7, n.2, p.241-252, 1991.

BUFFINGTON, E.D.; COLLIER, R.J.; CNATON, G.H. Shade management systems to reduce heat stress for dairy cows in hor humidt climate. Transaction of the American Society of Agricultural Engineering, v.26, n.8, p.1798-1802, 1983.

BUFFINGTON, D.E.; COLAZZO-AROCHO, A.; CATON, G.H., et al. Black globe humidity comfort index (BGHI) as comfort equation for dairy cows. Transaction of the
American Society Agricultural Engineering, v.24, n.4, p.711-714, 1981.

ESMAY, M.L.. Principles of animal environment. West Port: Avi Publishing, 1979. 325p.

HAFEZ, E.S.E. The behaviour of domestic animals. 2.ed. London: Bailiere Tindal, 1975. 436p.

HEAD, H.H. Management of dairy cattle in tropical and subtropical environments. In: CONGRESSO BRASILEIRO DE BIOMETEOROLOGIA, 2., 1995, Jaboticabal. Anais... Jaboticabal: SBBiomet, p.26-68. 1995.

HUBER, J.T.. Alimentação de vacas de alta produção sob condições de stress térmico. Bovinocultura leiteira. Piracicaba: Fundação de Estudos Agrários "Luiz de Queiroz", 1990. p.33-48.

INGRAM, D.L. Thermoregulatory behavior in pigs. Proceddings. In: WORLD CONGRESS ETHOLOGY APPLIED ZOOTECHNY, 1., 1978, Madrid. Proceedings... Madrid: 1978. p.137-141.

JOHNSON, H.D. Environmental management of cattle to minimize the stress of climatic change. International Journal of Biometeorology, v.24, n.1, p.65-78, 1980.

JOHNSON, K.G. Shading behaviour of sheep: preliminary studies of its relation to thermoregulation, feed and water intakes, and metabolic rates. Australian Journal Research, v.38, n.4, p.587-596, 1987.

RASGDALE, A.C. Environmental physiology with special reference to domestic animal, influence of increasing of temperature $40^{\circ}$ to $105^{\circ} \mathrm{F}$ on milk production in Brown Swiss cows, and on feed and water comsumption and body wieght in Brown Swiss and Brahman cows and heifers. Missouri Agiculture Research Bulletin, n.471, 1961.

SAS INSTITUTE. Statistical analysis system: realease 6.08, (software). Cary: 1992. 620p.

TINÔCO, I.F.F. Resfriamento adiabático evaporativo na produção de frangos de corte. Viçosa, MG: Universidade Federal de Viçosa, 1988. 92p. Dissertação (Mestrado em Engenharia Agrícola) - Universidade Federal de Viçosa, 1988.

THOM, E.C. Cooling degress: day air-conditioning, heating and ventilating. Transaction of the American Society of Heating, v.55, n.7, p.65-72, 1958.

R. Bras. Zootec., v.31, n.5, p.2036-2042, 2002

Recebido em: 13/07/01

Aceito em: 31/05/02 